\title{
Relationship of Tridimensional Personality Questionnaire Scores and Smoking Variables in Female and Male Smokers
}

\author{
Cynthia S. Pomerleau \\ Ovide F. Pomerleau \\ Karen A. Flessland \\ Susan M. Basson \\ Behavioral Medicine Research Laboratory, Department of Psychiatry \\ University of Michigan
}

\begin{abstract}
The Tridimensional Personality Questionnaire (TPQ) was developed by Cloninger (1986) to measure heritable variation in three patterns of response to environmental stimuli: novelty seeking, harm avoidance, and reward dependence. Cloninger (1987) used the TPQ to identify two types of alcoholism: Type I (low novelty seeking, high harm avoidance and reward dependence; both male and female) and Type 2 (high novelty seeking, low harm avoidance and reward dependence; predominantly male). To determine whether characteristic patterns exist in smokers, we administered the TPQ to 119 female and 121 male smokers, along with the Fagerstrom Tolerance Questionnaire (FTQ, a measure of nicotine dependence), the Russell Motives for Smoking Questionnaire (RMSQ), and the Spielberger StateTrait Anxiety Inventory (STAI/trait).

Compared with a normative sample, our sample exhibited elevated scores on the Novelty-Seeking scale; female smokers were somewhat overrepresented in the highest quartile of the Harm-Avoidance scale; both genders tended to be clustered in the lower quartiles of the Sentimentality-Attachment-Dependence subscale of the Reward-Dependence scale and in the highest quartile of the Persistence subscale. Female smokers showed a significant positive association between Harm Avoidance and FTQ scores, and Harm Avoidance was positively correlated with several RMSQ factors (including Addictive smoking) in both genders. These findings suggest that the likelihood of becoming a smoker may be a function of novelty seeking and reward dependence, whereas degree of dependence or addiction once the habit is entrained may be linked to harm avoidance. Our observations establish the potential utility of the $T P Q$ as a tool for examining environmental and heritable variation in smoking behavior and may contribute to improved strategies for prevention and treatment of smoking.
\end{abstract}

Support for this research was provided by National Cancer Institute grant CA 42730 and NIDA grant DA 06529 to the second author. We are grateful to T.F. Przybeck for supplying means and quartile cutoffs for the TPQ scoring system used in this study. We also wish to thank C. Robert Cloninger and Andrew Heath for their helpful comments on an earlier draft of this article.

Correspondence and requests for reprints should be sent to Cynthia S. Pomerleau, Behavioral Medicine Program, University of Michigan Department of Psychiatry, Riverview Medical Building, 900 Wall Street, Ann Arbor, MI 48105. 
Hughes (1986), in a review of adoption, association, family, trait-marker, and twin studies, concluded that smoking, like alcohol use, is influenced by heritable factors. Swan, Carmelli, Rosenman, Fabsitz, and Christian (1990) suggested that the heritability of smoking exceeds that of alcohol consumption. As Hughes observed, genotype could affect smoking either via personality or via differences in response to nicotine between smokers and nonsmokers. Eysenck's personality scales, for example, have been used in a number of prospective studies, and high scores on the Neuroticism and/or Extroversion scales have been found to predict both acquisition and maintenance of smoking (Cherry \& Kiernan, 1976; Eysenck \& Eaves, 1980). Zuckerman, Ball, and Black (1990) likewise reported that college undergraduates with high scores on the Sensation-Seeking scale are significantly more likely to be smokers than low scorers. Another possibility, not mentioned by Hughes, is that the degree to which the effects of nicotine are reinforcing under different circumstances may be inherited, that is, genetic determination of smoking motivation.

The Tridimensional Personality Questionnaire (TPQ; Cloninger, 1986; Cloninger, 1988; Cloninger, Pryzbeck, \& Svrakic, 1991) represents a systematic attempt not only to assess personality structure but to link it to its neuroregulatory substrates. It was designed to measure heritable variation in three patterns of response to specific environmental stimuli, characterized as follows:

1. "Novelty-seeking is hypothesized to be a heritable tendency toward intense exhilaration or excitement in response to novel stimuli or cues for potential rewards or potential relief of punishment, which leads to frequent exploratory activity in pursuit of potential rewards as well as active avoidance of monotony and potential punishment" (Cloninger, 1986, pp. 574-575). This trait may reflect degree of behavioral activation, which is thought to be modulated by dopaminergic activity.

2. "Harm-avoidance is a hereditable tendency to respond intensely to signals of aversive stimuli, thereby learning to inhibit behavior to avoid punishment, novelty, and frustrative nonreward" (Cloninger, 1986, p. 575). People high in this trait are likely to be characterized as "anxious." Harm avoidance may reflect variations in the brain's behavioral inhibition system, which is thought to be modulated by serotonergic activity.

3. "Reward-dependence is hypothesized to involve variation in behavioral maintenance or resistance to extinction of previously rewarded behavior. . . . Norepinephrine seems to satisfy the characteristics required of the major neuromodulator for this system and may play a critical role in the learning of new paired associations" (Cloninger, 1987, p. 414).

The TPQ has been used to characterize and predict a variety of psychiatric disorders (e.g., obsessive compulsive disorder [OCD]; Pfohl, Black, Noyes, Kelley, \& Blum, 1990). Cloninger (1987) used the TPQ to identify two distinct types of alcoholism: Type 1 (low novelty seeking, high harm avoidance and reward dependence; late onset, usually after an extended period of heavy drinking that is socially encouraged), and type 2 (high novelty seeking, low harm 
avoidance and reward dependence; early onset independent of external circumstances; drinking often associated with antisocial behavior). Type 1 alcoholics include both males and females; the Type 2 pattern is predominantly male.

Among the various approaches to personality assessment, Cloninger's strategy seems well adapted to analyzing the initiation and maintenance of drug selfadministration, which involves the use of substances that may modify mood or arousal level by acting at the receptor level to modify the bioavailability of relevant neuroregulators (e.g., Pomerleau \& Pomerleau, 1984). Accordingly, we routinely administered the TPQ to smokers who were recruited to participate either in laboratory investigations or in clinical trials of pharmacological agents to promote smoking cessation, along with questionnaires that assessed degree of dependence and motives for smoking. After 240 smokers had completed the TPQ, we attempted to determine (1) whether scores for any TPQ scale were associated with degree of dependence as measured by the Fagerstrom Tolerance Questionnaire (FTQ); and (2) whether TPQ scales could be used to differentiate "types" of smokers as determined by the Russell Motives for Smoking Questionnaire (RMSQ), in a way that might be comparable to the alcoholic typology discerned by Cloninger. Using Cloninger's norms, we also attempted to determine whether there might be a particular "profile" that could be said to characterize smokers in general.

\section{METHODS}

\section{Subjects}

Subjects were 240 smokers ( 119 female and 121 male) who were recruited to participate either in laboratory investigations ( 52 female, 65 male) or in clinical trials of pharmacological agents to promote smoking cessation (67 female, 56 male) in the Behavioral Medicine Program, University of Michigan Medical Center. Abuse of alcohol or other drugs, psychiatric illness, or any serious medical condition were exclusion criteria for all studies in which either subjects or patients participated.

\section{Procedure}

All smokers enrolling in laboratory investigations or clinical trials were given a packet of baseline questionnaires to fill out prior to their participation. These packets varied slightly depending on the study or treatment program in which subjects were involved (substitutes, updates, and deletions were made from time to time). Included in the analysis here were all those subjects $(N=240)$ who had provided both TPQ and FTQ data. The majority also had filled out the RMSQ $(n=216)$ and the trait section of the State-Trait Anxiety Inventory (STAI/trait; $n=230$ ). Because a comparison between the personality attributes of alcoholics and those of smokers was intended, it seemed important to consider the possibility that the drinking behavior of our subjects might influence or confound our findings; although systematic data on alcohol intake were not collected, it 
was possible to dichotomize 228 of the 240 subjects on the basis of whether or not they consumed two or more alcoholic drinks per day (a drink being defined as one beer, one glass of wine, or one shot of liquor).

Using normative data from a sample of white adults (326 males, mean age $=$ $43.2 \pm 16.4 S D$; and 350 females; $M=45.3 \pm 18.1 S D$ ) unselected for smoking status (Cloninger et al., 1991), the quartile into which scores of each subject from our sample fell was calculated, and chi-square analyses were conducted to test for goodness of fit with respect to Cloninger's norms. (The scoring system used in our study was subsequently revised slightly by Cloninger and colleagues.) To determine whether the TPQ could be used to predict degree of nicotine dependence, we examined the relationship between TPQ scale scores and FTQ scores (Fagerstrom, 1978), a frequently used index of nicotine dependence (Hughes, 1984; Pomerleau, Majchrzak, \& Pomerleau, 1989; Pomerleau, Pomerleau, Majchrzak, Kloska, \& Malakuti, 1990). To determine whether smokers of different types were characterized by distinct patterns or combinations of TPQ scale scores, we examined the relationship between TPQ and the six factors of Russell's smoker classification scheme (Russell, Peto, \& Patel, 1973), slightly modified. (Only the three questions loading the highest for each factor were used, with the possible scores for each factor ranging from 0 to 9; for Factor VI, the wording of Russell's Question 10 was changed to read, "I've found a cigarette in my mouth without recalling putting it there.") 'The trait scale of the STAI (Spielberger, Gorsuch, Lushene, Vagg, \& Jacobs, 1983) was used to help validate the link between harm avoidance and trait anxiety. Males and females were examined separately because of expected differences (based on Cloninger's norms) in TPQ scores. Because multiple statistical tests were planned, a conservative alpha of .01 was set as the criterion for significance to reduce the likelihood of Type I error.

\section{RESULTS}

Patients participating in clinical trials were significantly older than laboratory subjects $[40.4 \pm .7$ vs. $31.1 \pm .6 ; t(237)=-9.66, p<.001]$ and smoked significantly more cigarettes per day $[32.8 \pm 1.1$ vs. $25.1 \pm .8 ; t(237)=-5.78$, $p<.001]$. Because there were no significant differences between patients and laboratory subjects on any of the TPQ scales for either males or females, nor on alcohol intake, data for these two subsamples were pooled. Female and male subjects did not differ significantly with respect to age (35.3 \pm 9.0 years vs. 36.5 \pm 8.4 years $)$ or cigarettes per day $(27.4 \pm 9.1$ for the females vs. $30.5 \pm 12.4$ for the males). A significantly larger percentage of male smokers (19\%; 21/112) than female smokers $(8 \% ; 9 / 116)$ consumed two or more drinks per day, $\chi^{2}(1, N=$ $228)=5.10, p<.05$. Using quartile scores to classify our subjects into Type 1 (Novelty-Seeking in the lowest quartile, Harm-Avoidance and Reward-Dependence in the highest quartile) and Type 2 (Novelty-Seeking in the highest quartile, Harm-Avoidance and Reward-Dependence in the lowest quartile) patterns as seen in alcoholics, 1 female could be classified as Type 1 and 10 male subjects could be classified as Type 2 . Of these 11 classifiable subjects, 8 reported drink- 
ing fewer than two drinks per day and 3 (all male Type 2) reported drinking two or more drinks per day.

Table 1 shows mean TPQ scores for our sample of female and male smokers and for Cloninger's (1987) sample of white adults (data supplied by T.F. Przybeck, personal communication, December 16, 1991); because Cloninger et al. (1991) suggested that the Reward-Dependence scale may not be unidimensional, we also included scores for its two subscales (Sentimentality-AttachmentDependence and Persistence). The distribution of our subjects by quartile, with respect to Cloninger's norms, is shown in Figures 1 and 2. To determine whether the distribution of our subjects differed significantly from the quartile distribution that would be predicted by Cloninger's norms, we conducted a series of goodness-of-fit tests. (Because Novelty-Seeking shows a weak but reliable negative correlation with age, with scores declining by about a point per decade, and because our sample had a mean age 8-10 years younger than Cloninger's respondents, the percentages per normative quartile for this scale were recalculated prior to further analysis, using cutoffs a point higher than those supplied by Cloninger; $46 \%$ of the females and $41 \%$ of the males remained in the highest quartile.) For both males and females, more of our subjects had Novelty-Seeking scores in the highest quartile than would be expected, even when adjusted for age differences [females, $\chi^{2}(3, N=119)=28.86, p<.001 ;$ males, $\chi^{2}(3, N=121)$ $=28.45, p<.001]$. For Harm-Avoidance, females clustered in the highest quartile, $\chi^{2}(3, N=119)=11.52, p<.01$, but males did not, $\chi^{2}(3, N=121)=6.97$, n.s. Reward-Dependence showed clustering in the lowest quartile for males, $\chi^{2}$ $(3, N=121)=20.13, p<.001$, but not for females, $\chi^{2}(3, N=119)=6.02$, n.s. For both males and females, the two Reward-Dependence subscales showed opposing directions, with females clustering in the lower quartiles, $\chi^{2}(3, N=$ $119)=13.54, p<.005$, and males in the lowest quartile, $\chi^{2}(3, N=121)=70.58$,

Table 1. TPQ Scores for Pomerleau et al. (1991) Subjects (Smokers) Compared with Normative Data for General Population of White Adults (Cloninger et al. [1987])

\begin{tabular}{lcc}
\hline & $\begin{array}{c}\text { Pomerleau et al. (1991) } \\
\text { Subjects } \\
M \pm S D\end{array}$ & $\begin{array}{c}\text { Cloninger (1991) } \\
\text { Norms }\end{array}$ \\
\hline Females & $(n=119)$ & $M \pm S D$ \\
Novelty-Seeking & $16.8 \pm 5.2$ & $(n=350)$ \\
Harm-Avoidance & $14.8 \pm 6.5$ & $13.0 \pm 4.9$ \\
Reward-Dependence & $22.8 \pm 4.5$ & $12.8 \pm 6.0$ \\
Sentimentality & $14.6 \pm 3.8$ & $22.3 \pm 3.9$ \\
Persistence & $8.2 \pm 2.3$ & $15.7 \pm 3.3$ \\
Males & $(n=121)$ & $6.5 \pm 2.0$ \\
Novelty-Seeking & $17.2 \pm 5.0$ & $(n=326)$ \\
Harm-Avoidance & $11.5 \pm 6.3$ & $13.7 \pm 5.2$ \\
Reward-Dependence & $19.3 \pm 5.0$ & $10.5 \pm 5.9$ \\
Sentimentality & $11.5 \pm 4.1$ & $20.6 \pm 4.5$ \\
Persistence & $7.7 \pm 2.5$ & $14.1 \pm 3.7$ \\
\hline
\end{tabular}


NOVELTY-SEEKING

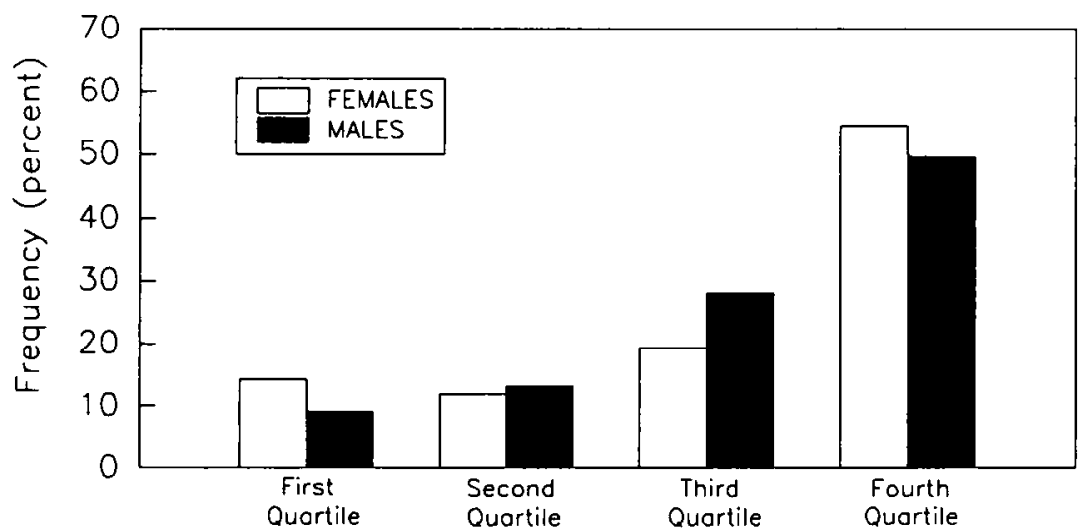

HARM-AVOIDANCE

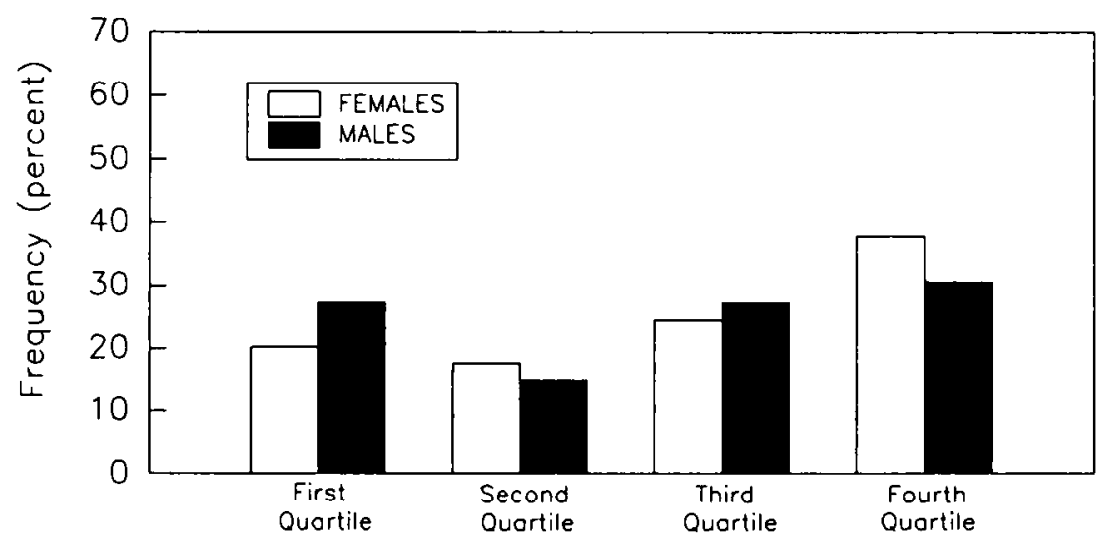

REWARD-DEPENDENCE

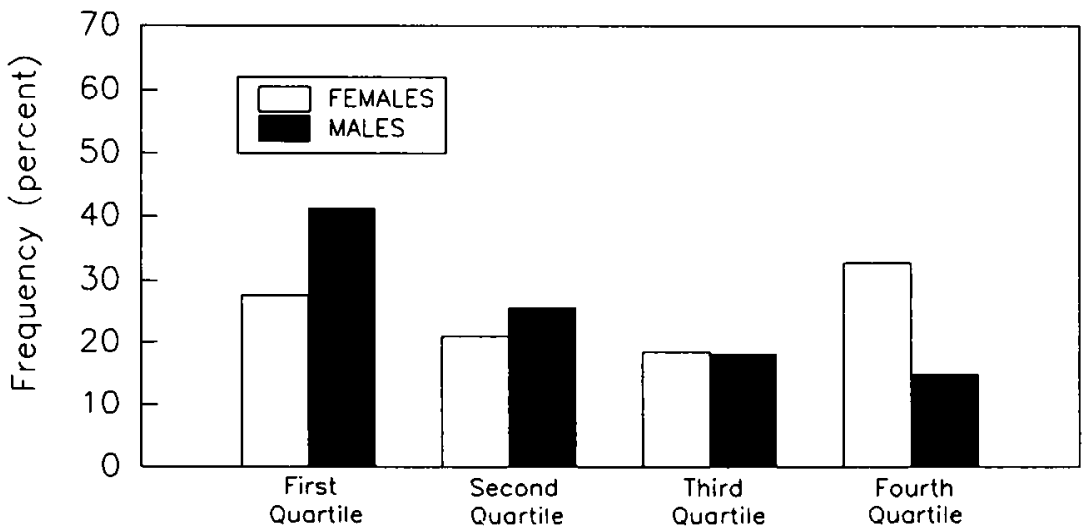

Figure 1. Distribution of smoking sample (\%) by quartile in normative sample (T. $R$. Pryzbeck, personal communication, 1991) for Novelty-Seeking, Harm-Avoidance, and Reward-Dependence scales, TPQ. 

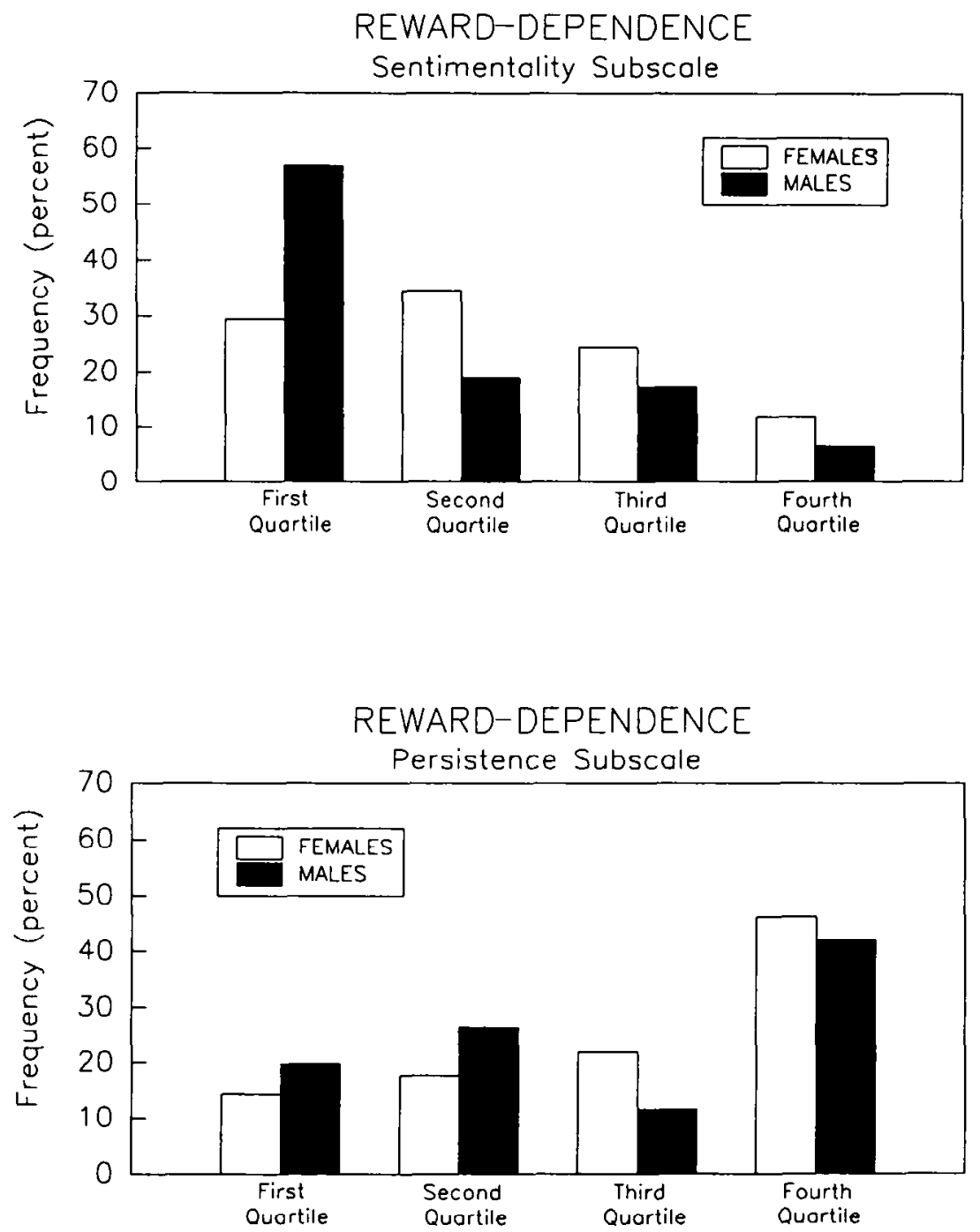

Figure 2. Distribution of smoking sample (\%) by quartile in normative sample (T. R. Pryzbeck, personal communication, 1991) for Reward-Dependence subscales (Subscale 1: Sentimentality-Dependence-Attachment; Subscale 2: Persistence), TPQ.

$p<.001$, of the Sentimentality-Attachment-Dependence subscale but in the highest quartile of the Persistence subscale [females, $\chi^{2}(3, N=119)=29.92$, $p<.001$; males, $\left.\chi^{2}(3, N=121)=24.35, p<.001\right]$.

Table 2 shows STAI/trait, FTQ, and RMSQ scores for all subjects. STAI/trait scores in our sample are similar to, though slightly higher than, norms for working adults in the 19-39 age range (35.55 \pm 9.76 for males and $36.15 \pm 9.53$ for females; Spielberger et al., 1983). FTQ scores characterize our sample as fairly heavy smokers. No normative data exist for the modified RMSQ. Females in our sample did not differ significantly from males with respect to either 
Table 2. Spielberger State-Trait Anxiety Inventory/Trait (STAI), Fagerstrom Tolerance Questionnaire (FTQ), and Russell Motives for Smoking Questionnaire (RMSQ-modified) Scores

\begin{tabular}{lll}
\hline & $\begin{array}{c}\text { Females } \\
M \pm S D\end{array}$ & \multicolumn{1}{c}{$\begin{array}{c}\text { Males } \\
M\end{array}$} \\
& $(n=114)$ & $(n=116)$ \\
& $37.6 \pm 10.3$ & $37.6 \pm 8.7$ \\
STAI/Trait & $(n=119)$ & $(n=121)$ \\
& $7.7 \pm 1.5$ & $7.4 \pm 1.5$ \\
FTQ $(1-11)$ & & \\
& $(n=119)$ & $(n=97)$ \\
RMSQ & $4.9 \pm 2.1$ & $4.3 \pm 2.1$ \\
Stimulation & $3.8 \pm 1.8$ & $3.5 \pm 1.7$ \\
Indulgent & $3.7 \pm 2.3^{\circ}$ & $2.6 \pm 2.1^{\circ}$ \\
Psychosocial & $2.3 \pm 1.6$ & $1.9 \pm 1.6$ \\
Sensorimotor & $6.5 \pm 1.7^{*}$ & $5.4 \pm 1.9^{*}$ \\
Addictive & $2.1 \pm 2.2$ & $2.2 \pm 2.3$ \\
Automatic & &
\end{tabular}

$* p<.001$.

Anxiety, as measured by the SIAI/trait, or Nicotine Dependence, as measured by the FTQ. Scores on two of the six RMSQ factors-Psychosocial and Addictive-differed significantly between the genders.

STAI/trait scores were highly correlated with Harm-Avoidance scores for both females $(r=.54, p<.001)$ and males $(r=.60, p<.001)$; no direct correlation, however, was observed between STAI/trait and FTQ scores. Table 3 shows correlations for TPQ scales and the two Reward-Dependence subscales with FTQ scores and RMSQ factors for females and for males.

\section{DISCUSSION}

The personality profile that emerges from the analyses of the smokers in our sample is as follows: First, smokers of both genders are more likely than the population as a whole to be high in Novelty-Seeking. Female smokers, but not male smokers, were somewhat more likely to score high in Harm-Avoidance. Male smokers tend to cluster in the lowest quartile for Reward-Dependence overall, unlike female smokers, who are distributed more nearly in accord with population norms. Both genders, however, scored low in the SentimentalityAttachment-Dependence subscale and high in the Persistence subscale. Second, in females only, Harm-Avoidance is positively correlated with degree of dependence as measured by the FTQ; although a relationship between Harm-Avoidance and Nicotine Dependence (as measured by FTQ scores) did not emerge for males, the significant correlation of Harm-Avoidance with the RMSQ Addictive and several other factors for males as well as females suggests that harm avoidance may play a role in addictive smoking in males as well. Our attempts to associate RMSQ factors with discernible patterns or combinations of high and/or 
Table 3. Correlations of TPQ Scores and Reward-Dependence Subscale Scores with FTQ and RMSQ-Modified Scores in Females and Males

\begin{tabular}{|c|c|c|c|c|c|}
\hline & $\begin{array}{l}\text { TPQ-NS } \\
\text { Novelty } \\
\text { Seeking }\end{array}$ & $\begin{array}{c}\text { TPQ-HA } \\
\text { Harm } \\
\text { Avoidance }\end{array}$ & $\begin{array}{c}\text { TPQ-RD } \\
\text { Reward- } \\
\text { Dependence }\end{array}$ & $\begin{array}{c}\text { TPQ-RD } \\
\text { Sentimentality, } \\
\text { etc. }\end{array}$ & $\begin{array}{c}\text { TPQ-RD } \\
\text { Persistence }\end{array}$ \\
\hline \multicolumn{6}{|l|}{ Females } \\
\hline $\begin{array}{l}\text { FTQ }(n=119) \\
\text { RMSO }(n=119)\end{array}$ & -.1084 & $.3251 * * * *$ & -.0823 & .1245 & .0447 \\
\hline Stimulation & -.0469 & $.2423 * *$ & .1307 & .0955 & .1062 \\
\hline Indulgent & -.1118 & -.0425 & -.0739 & -.0875 & .0121 \\
\hline Psychosocial & -.0097 & .1735 & .1267 & .1319 & .0343 \\
\hline Sensorimotor & .1740 & -.0473 & .0121 & .0619 & -.0870 \\
\hline Addictive & -.0820 & $.2820 * * *$ & .1730 & .1132 & .1583 \\
\hline Automatic & -.1320 & .1098 & -.0978 & -.1405 & .0533 \\
\hline \multicolumn{6}{|l|}{ Males } \\
\hline $\begin{array}{l}\text { FTQ }(n=121) \\
\operatorname{RMSQ}(n=97)\end{array}$ & .0338 & .0407 & -.0271 & .0113 & .0732 \\
\hline Stimulation & -.0398 & $.2420^{*}$ & -.0235 & -.0402 & .0184 \\
\hline Indulgent & -.0305 & -.0598 & -.0041 & -.0030 & -.0036 \\
\hline Psychosocial & -.0298 & $.3795 * * * *$ & .0648 & .0951 & -.0239 \\
\hline Sensorimotor & .0727 & $.2128^{*}$ & -.0618 & -.0249 & -.0881 \\
\hline Addictive & -.0112 & $.4343 * * * *$ & -.0045 & -.0067 & .0020 \\
\hline Automatic & .1488 & $.2352 * * *$ & -.0218 & -.0646 & .0630 \\
\hline
\end{tabular}

low scores on the three TPQ scales produced nothing comparable to Cloninger's Type 1 and Type 2 alcoholics, though possibly such patterns would emerge in a larger sample.

Our findings for the Novelty-Seeking scale in an adult sample tends to confirm Zuckerman et al.'s (1990) suggestion that this trait is more marked in smokers than in the general population, even when cutoffs were adjusted to allow for age differences between our sample and that of Cloninger et al. (1991). Indeed, because around 29\% of American adults reported smoking in 1987 (USDHHS, 1989), when the normative sample was tested, comparison with Cloninger's (1991) unselected sample may well underestimate the differences between smokers and nonsmokers. If, as Cloninger (1987) argued, individuals with high Novelty-Seeking scores are particularly responsive to potential rewards or their conditioned signals, they might be expected to be particularly susceptible to tobacco advertising and to the blandishments of nicotine as delivered by smoking (which repeatedly produces small but reliable reinforcing effects and promotes a self-administration schedule highly conducive to conditioning).

Because the subscales for Reward-Dependence produced discordant patterns of distribution in our sample, the subscale scores may be of greater interest than the overall factor score. Our subjects appeared to cluster in the lower half (for males, the lowest quartile) of the Sentimentality-Attachment-Dependence subscale, possibly accounting for indifference towards social pressures to quit. By contrast, both genders were well represented in the highest quartile of the Per- 
sistence subscale. To the extent that high Persistence scores represent resistance to extinction of previously rewarded behavior (Cloninger, 1987), an association of Persistence scores with smoking behavior has considerable face validity, as the statistics for treatment of smoking attest.

Although females (but not males) showed some evidence of overrepresentation in the highest quartile for Harm-Avoidance, the most remarkable observation about this TPQ scale was its apparent association with degree of addiction. Cloninger (1988) presented evidence that high Harm-Avoidance scores are associated with cognitive anxiety, a finding supported in our own database by the strong association between STAI/trait and Harm-Avoidance scores in both males and females. Cloninger (1987) further indicated that some males and most females who are dependent on alcohol tend to have personalities that are characterized by cognitive anxiety. Our findings suggest that a similar pattern may prevail for highly dependent smokers, though the full Type 1 alcoholic constellation (low novelty seeking, high harm avoidance, high reward dependence) was not observed, and though the STAI/trait did not predict F"TQ score directly. Nicotine's documented anxiolytic effects (Pomerleau, 1986) suggest the possibility that, at least in some highly dependent smokers, smoking may be a pharmacological coping response (Pomerleau \& Pomerleau, 1984) or even a form of "self-medication." A recent report (Pfohl et al., 1990) of significantly elevated Harm-Avoidance scores in individuals with $\mathrm{OCD}$, as compared with normal controls, also may shed light on our findings, insofar as drug-craving and drug-seeking behavior in drug-dependent individuals have been likened respectively to obsessions and compulsions comparable to those observed in OCD (Caetano, 1985).

A few caveats are in order. First, because our study did not include a nonsmoking comparison group tested under conditions similar to those under which our smoking data were collected, our inferences regarding smokers are based on normative data in an unselected sample and therefore must be regarded as tentative. Second, though we found no evidence that our findings for the TPQ were in any way secondary to drinking behavior, our measures were too imprecise, and our sample too restricted with respect to drinking patterns, to rule out this possibility altogether. Third, it must be pointed out that the mean FTQ score for our sample was relatively high, and different results might have been found in a broader sample of smokers (i.e., a sample that included chippers as well as a larger number of less dependent smokers). Finally, some of the subjective instruments used in this study suffer from lack of adequate validity and reliability testing (though unfortunately, superior alternatives were not available).

Despite the preliminary nature of our observations, we believe that they establish the TPQ as a potentially useful tool for understanding environmental and heritable variations in smoking behavior. If our findings hold up in larger samples that include nonsmokers as well as smokers, it may eventually be possible to develop prediction equations in which likelihood of becoming a smoker is expressed as a function of Novelty-Seeking and of the Reward-Dependence subscales, whereas degree of dependence or addiction once the habit is entrained is 
linked to Harm-Avoidance. Such a conclusion would suggest that the inherited and environmental factors that institute and/or maintain smoking differ from those that govern degree of dependence; this inference is in fact consistent with the finding, based on analyses of data from a U.S. twin study, that separate genetic and environmental factors are involved in the determination of initiation of smoking, on the one hand, and the amount smoked by those who become smokers, on the other (J. Meyer and A. Heath, personal communication, December 23, 1991). Further twin studies and prospective studies of children who have not reached smoking age, however, will be needed to support and extend our understanding of these phenomena; direct tests in smokers of Cloninger's (1987) neuroregulatory hypotheses both at baseline and in response to nicotine challenge will be needed as well, and could result in the identification of biochemical markers for susceptibility to smoking initiation or dependence. Thus, our findings, if confirmed and extended as previously described, may increase the understanding of smoking as well as contribute to the development of improved strategies for prevention and intervention.

\section{REFERENCES}

Caetano, R. (1985). Alcohol dependence and the need to drink: A compulsion? Psychological Medicine, $15,463-469$.

Cherry, N., \& Kiernan, K. (1976). Personality scores and smoking behavior: A longitudinal study. British Joumal of Preventive and Social Medicine, 30, 123-131.

Cloninger, C.R. (1986). A systematic method for clinical description and classification of personality variants: A proposal. Archives of General Psychiatry, 44, 573-588.

Cloninger, C.R. (1987). Neurogenetic adaptive mechanisms in alcoholism. Science, 236, 410-416.

Cloninger, C.R. (1988). A unified biosocial theory of personality and its role in the development of anxiety states. Psychiatric Developments, 3, 167-226.

Cloninger, C.R., Przybeck, T.R., \& Svrakic, D.M. (1991). The Tridimensional Personality Questionnaire: U.S. normative data. Psychological Reports, 69, 1047-1057.

Eysenck, H.J., \& Eaves, E.J. (1980). The causes and effects of smoking. London: Maurice Temple Smith.

Fagerstrom, K.-O. (1978). Measuring degree of physical dependence to tobacco smoking with reference to individualization of treatment. Addictive Behaviors, 3, 235-24l.

Hughes, J.R. (1984). Identification of the dependent smoker: Validity and clinical utility. Behavioral Medicine Abstracts, 3, 202-204.

Hughes, J.R. (1986). Genetics of smoking: A brief review. Belavior Therapy, 17, 335-345.

Pfohl, B., Black, D., Noyes, R., Kelley, M., \& Blum, N. (1990). A test of the tridimensional personality theory: Association with diagnosis and platelet imipramine binding in obsessive-compulsive disorder. Biological Psychiatry, 28, 41-46.

Pomerleau, C.S., Majchrzak, M.J., \& Pomerleau, O.F. (1989). Nicotine dependence and the Fagerstrom Tolerance Questionnaire: A brief review. Journal of Substance Abuse, 1, 471-477.

Pomerleau, C.S., Pomerleau, O.F., Majchrzak, M.J., Kloska, D.D., \& Malakuti, R. (1990). Relationship between Nicotine Tolerance Questionnaire scores and plasma cotinine. Addictive Behaviors, 15 , $73-80$.

Pomerleau, O.F. (1986). Nicotine as a psychoactive drug: Anxiety and pain reduction. Psychopharmacology Bulletin, 22, 865-869.

Pomerleau, O.F., \& Pomerleau, C.S. (1984). Neuroregulators and the reinforcement of smoking: Towards a biobehavioral explanation. Neuroscience $\mathcal{E}$ Biobehavioral Reviews, 8, 503-513.

Russell, M.A.H., Peto, J., \& Patel, U.A. (1973). The classification of smoking by factorial structure of motives. Journal of the Royal Statistical Society, 137, 313-333. 
Spielberger, C.D., Gorsuch, R.L., Lushene, R., Vagg, P.R., \& Jacobs, G.A. (1983). Manual for the StateTrait Anxiety Inventory. Palo Alto, CA: Consulting Psychologists Press.

Swan, G.E., Carmelli, D., Rosenman, R.H., Fabsitz, R.R., \& Christian, J.C. (1990). Smoking and alcohol consumption in adult male twins: Genetic heritability and shared environmental influences. Journal of Substance Abuse, 2, 39-50.

United States Department of Health and Human Services (USDHHS). (1989). Reducing the Health Consequences of Smoking: 25 Years of Progress. A Report of the Surgeon General (Public Health Service, Centers for Disease Control, Center for Chronic Disease Prevention and Health Promotion, Office on Smoking and Health; DHHS Publication No. CDC 89-8411). Washington, DC: U.S. Government Printing Office.

Zuckerman, M., Ball, S., \& Black, J. (1990). Influences of sensation seeking, gender, risk appraisal, and situational motivation on smoking. Addictive Behaviors, 15, 209-220. 